\title{
Manifestações orais da Sífilis: uma revisão da literatura
}

\author{
Syphilis oral manifestations: a literature review \\ Manifestaciones orales de la sífilis: revisión de la literatura
}

Ana Luiza Castro Binda ORCID: https://orcid.org/0000-0002-6664-2538 Escola São Francisco de Assis, Brasil

E-mail: aninha_sgp@hotmail.com

Anne Caroline Silva Freire de Sá ORCID: https://orcid.org/0000-0003-3860-1373 Centro Universitário Tabosa de Almeida, Brasil

E-mail: annecarolinefreiree@gmail.com

Amanda Gonçalves Franco

ORCID: https://orcid.org/0000-0003-0983-7539

Universidade de Itaúna, Brasil

E-mail: amandagfranco38@gmail.com

Thalita Oliveira da Silva Borba

ORCID: https://orcid.org/0000-0001-5539-7744

Centro Universitário Tabosa de Almeida, Brasil

E-mail: thalita.borba00@gmail.com

Hana Yasmim Marques Silva de Souza

ORCID: https://orcid.org/0000-0003-0128-7720

Centro Universitário Tiradentes de Pernambuco, Brasil

E-mail: hanayasmim@hotmail.com

João Wictor Leandro da Silva

ORCID: https://orcid.org/0000-0002-9941-147X

Universidade Tiradentes de Pernambuco, Brasil E-mail: wictor_joao@hotmail.com

Fernanda da Rosa Savi

ORCID: https://orcid.org/0000-0001-9500-9988

Universidade do Extremo Sul Catarinense, Brasil E-mail: fefasavi@gmail.com

Harysson Costa Melo

ORCID: https://orcid.org/0000-0001-7427-7975

Universidade de Fortaleza, Brasil

E-mail: haryssoncoslo@gmail.com

George Jose de Oliveira Filho

ORCID: https://orcid.org/0000-0001-7275-9612

Universidade Tiradentes, Brasil

E-mail: georgejose49@hotmail.com

Mateus Biola Melo Ferreira

ORCID: https://orcid.org/0000-0002-0380-5555

Universidade de Rio Verde, Brasil

E-mail: biolamateus@gmail.com

Edyssa Laryssa da Silva Ferreira de Araújo ORCID: https://orcid.org/0000-0002-4799-6330

Universidade Federal de Alagoas, Brasil

E-mail: edyssa.araujo@foufal.ufal.br

Matheus Almeida Ramalho

ORCID: https://orcid.org/0000-0002-5607-1313

Universidade Federal de Jataí, Brasil

E-mail: matheusramalho@discente.ufg.br

Marcela Nogueira dos Santos

ORCID: https://orcid.org/0000-0002-0554-6382

Faculdade de Odontologia do Recife, Brasil

E-mail: marcelaanogueira.santos@gmail.com

José Victor Lima Silva

ORCID: https://orcid.org/0000-0002-8539-5456

Centro Universitário Christus, Brasil

E-mail: victorlimasv@ hotmail.com

Bruna Peixoto Girard

ORCID: https://orcid.org/0000-0003-0233-666X

Centro Universitário Cesmac, Brasil

E-mail: brunapeixoto5@hotmail.com 


\author{
Jaqueline Lopes Reis \\ ORCID: https://orcid.org/0000-0003-1685-0336 \\ Universidade de Rio Verde, Brasil \\ E-mail: jaquelreis@hotmail.com
}

\begin{abstract}
Resumo
A sífilis consiste em uma doença infecciosa causada pelo Treponema Pallidum, normalmente por transmissão vertical, sendo conhecida como sífilis congênita, ou, ainda, sexual, chamada de sífilis adquirida. Além disso, a contaminação pode ocorrer de forma indireta, pela utilização de objetos contaminados, como agulhas de tatuagem ou transfusão sanguínea. É considerada um problema de saúde pública, devido ao seu alto grau de incidência, majoritariamente em países de baixa renda. O registro de casos da doença tem aumentado nos últimos vinte anos. Diante do exposto, este artigo possui como objetivo revisar a literatura acerca das manifestações bucais da sífilis. Para a construção deste trabalho foi feito um levantamento bibliográfico nas bases de dados SciVerse Scopus, Scientific Eletronic Library Online (Scielo), U.S. National Library of Medicine (PUBMED) e ScienceDirect, utilizando o gerenciador de referências Mendeley. Os artigos foram coletados no período de maio a agosto de 2021 e contemplados entre os anos de 2015 a 2021. A partir da revisão narrativa da literatura, observou-se que as manifestações orais decorrentes da sífilis podem ocorrer de diversas formas, a depender do seu estágio de infecção e evolução da doença. O papel do cirurgião dentista se torna importante para o diagnóstico dos pacientes infectados, para o sucesso do tratamento das lesões orais, melhorando seu prognostico e qualidade de vida.
\end{abstract}

Palavras-chave: Sífilis; Sífilis primária; Sífilis secundária; Sífilis terciária; Sífilis congênita.

\begin{abstract}
Syphilis is an infectious disease caused by Treponema Pallidum, usually by vertical transmission, known as congenital syphilis, or even sexual, called acquired syphilis. In addition, contamination can occur indirectly, through the use of contaminated objects, such as tattoo needles or blood transfusions. It is considered a public health problem due to its high degree of incidence, mostly in low-income countries. The record of cases of the disease has increased in the last twenty years. Given the above, this article aims to review the literature on the oral manifestations of syphilis. SciVerse Scopus, Scientific Electronic Library Online (Scielo), US National Library of Medicine (PUBMED) and ScienceDirect, with assistance from Mendeley. The articles were collected from May to August 2021 and covered between 2015 and 2021. From the review of the literature narrative, it was observed that oral manifestations resulting from syphilis can occur in different ways, depending on the its stage of infection and disease evolution. The role of the dental surgeon becomes important for the diagnosis of infected patients, for the successful treatment of oral lesions, improving their prognosis and quality of life.
\end{abstract}

Keywords: Syphilis; Primary syphilis; Secondary syphilis; Tertiary syphilis; Congenital syphilis.

\title{
Resumen
}

La sífilis es una enfermedad infecciosa causada por Treponema pallidum, generalmente por transmisión vertical, conocida como sífilis congénita, o incluso sexual, llamada sífilis adquirida. Además, la contaminación puede ocurrir indirectamente, mediante el uso de objetos contaminados, como agujas de tatuajes o transfusiones de sangre. Se considera un problema de salud pública por su alto grado de incidencia, principalmente en países de bajos ingresos. El registro de casos de la enfermedad se ha incrementado en los últimos veinte años. Dado lo anterior, este artículo tiene como objetivo revisar la literatura sobre las manifestaciones orales de la sífilis. Para la construcción de este trabajo, se realizó un levantamiento bibliográfico en las bases de datos SciVerse Scopus, Scientific Electronic Library Online (Scielo), U.S. National Library of Medicine (PUBMED) y ScienceDirect, con la ayuda de Mendeley. Los artículos fueron recolectados de mayo a agosto de 2021 y cubiertos entre los años 2015 a 2021 . A partir de la revisión narrativa de la literatura, se observó que las manifestaciones bucales derivadas de la sífilis pueden presentarse de diferentes formas, dependiendo de su etapa de infección y evolución de la enfermedad. El papel del cirujano dental se vuelve importante para el diagnóstico de los pacientes infectados, para el tratamiento exitoso de las lesiones bucales, mejorando su pronóstico y calidad de vida.

Palabras clave: Sífilis; Sífilis primaria; Sífilis secundaria; Sífilis terciaria; Sífilis congénita.

\section{Introdução}

A sífilis é uma doença infecciosa causada pelo Treponema Pallidum e que possui duas vias de transmissão. A transmissão vertical é chamada de sífilis congênita e a sífilis adquirida caracteriza-se por transmissão sexual. Além disso, a contaminação pode ocorrer de forma indireta, pela utilização de objetos contaminados, como agulhas de tatuagem ou transfusão sanguínea (Fregnani et al., 2017). 
Na sífilis adquirida, o agente infeccioso Treponema Pallidum penetra nos tecidos humano, através de pequenas abrasões durante a relação sexual, atingindo o sistema linfático regional e, a partir da disseminação teratogênica, se dissemina para todo o corpo. Quando o agente infeccioso se encontra no sangue da gestante, atravessa a barreira placentária, atingindo a corrente sanguínea do feto, ou ainda, pode haver a contaminação na passagem pelo canal do parto, chamada de sífilis congênita.

Habitualmente, a sífilis acomete todos os órgãos e sistema do corpo humano e, apesar dos avanças nas medidas terapêuticas, a doença ainda é considerada um problema de saúde pública, devido ao seu alto grau de incidência, majoritariamente em países de baixa renda. O registro de casos da doença tem aumentado nos últimos vinte anos (De Andrade et al., 2020).

As manifestações clínicas dependem do estágio da doença e evolução das lesões. O diagnóstico é dificultado, pois a condição pode se assemelhar com outras entidades patológicas e requer maior atenção por parte de médicos, cirurgiões dentistas e patologistas (Schuch et al., 2019).

Profissionais da saúde devem estar preparados para diagnosticarem, excluindo seus diagnósticos diferenciais, evitando diagnósticos equivocados, melhorando o prognostico e qualidade de vida do paciente. Diante do exposto, este artigo possui como objetivo revisar a literatura acerca das manifestações bucais da sífilis.

\section{Metodologia}

Refere-se a uma revisão integrativa de literatura, de caráter qualitativa. A revisão de literatura permite a busca aprofundada dentro de diversos autores e referenciais sobre um tema específico, nesse caso, as manifestações orais decorrentes da radioterapia de cabeça e pescoço (Pereira et al., 2018).

A fim de que haja direcionamento na pesquisa, delineou-se como questão norteadora: "quais são as manifestações orais decorrentes da infecção por Treponema Pallidum?".

\subsection{Busca Estratégica}

Para a construção deste artigo foi feito um levantamento bibliográfico nas bases de dados SciVerse Scopus, Scientific Eletronic Library Online (Scielo), U.S. National Library of Medicine (PUBMED) e ScienceDirect, utilizando o gerenciador de referências Mendeley. Os artigos foram coletados no período de maio a agosto de 2021 e contemplados entre os anos de 2015 a 2021.

A estratégia de pesquisa desenvolvida para identificar os artigos incluídos e avaliados para este estudo baseou-se nos descritores contidos na lista dos Descritores em Ciência da Saúde (DeCS) e suas combinações no idioma português e inglês: [(sífilis OR syphilis OR treponema pallidum) AND (primária OR primary OR secundária OR secondary OR terciária OR tertiary OR congênita OR congenital) AND (boca OR mouth OR oral OR manifestações clínicas OR clinical findings)].

\subsection{Critérios de inclusão e exclusão}

Considerou-se como critério de inclusão os artigos completos disponíveis na íntegra nas bases de dados citadas, nos idiomas inglês e português e relacionados com o objetivo deste estudo.

Os critérios de exclusão foram artigos incompletos, duplicados, resenhas, estudos in vitro e resumos.

\subsection{Seleção de estudos}

A estratégia de pesquisa baseou-se na leitura dos títulos para encontrar estudos que investigassem a temática da pesquisa. Caso atingisse esse primeiro objetivo, posteriormente, os resumos eram lidos e, persistindo na inclusão, era feita a 
leitura do artigo completo. Quando havia dúvida sobre a inclusão, o artigo era lido por outro autor e, a decisão de inclusão ou exclusão era tomada em consenso.

Na sequência metodológica foi realizada a busca e leitura na íntegra dos artigos pré-selecionados, os quais foram analisados para inclusão da amostra.

\section{Resultados e Discussão}

Com base na revisão de literatura feita nas bases de dados eletrônicas citadas, foram identificados 2678 artigos científicos, dos quais 255 estavam duplicados com dois ou mais índices. Após a leitura e análise do título e resumos dos demais artigos outros 2347 foram excluídos. Assim, 76 artigos foram lidos na integra e, com base nos critérios de inclusão e exclusão, apenas 24 artigos foram selecionados para compor este estudo. O fluxograma com detalhamento de todas as etapas de seleção está na Figura 1.

Figura 1 - Fluxograma de identificação e seleção dos estudos.

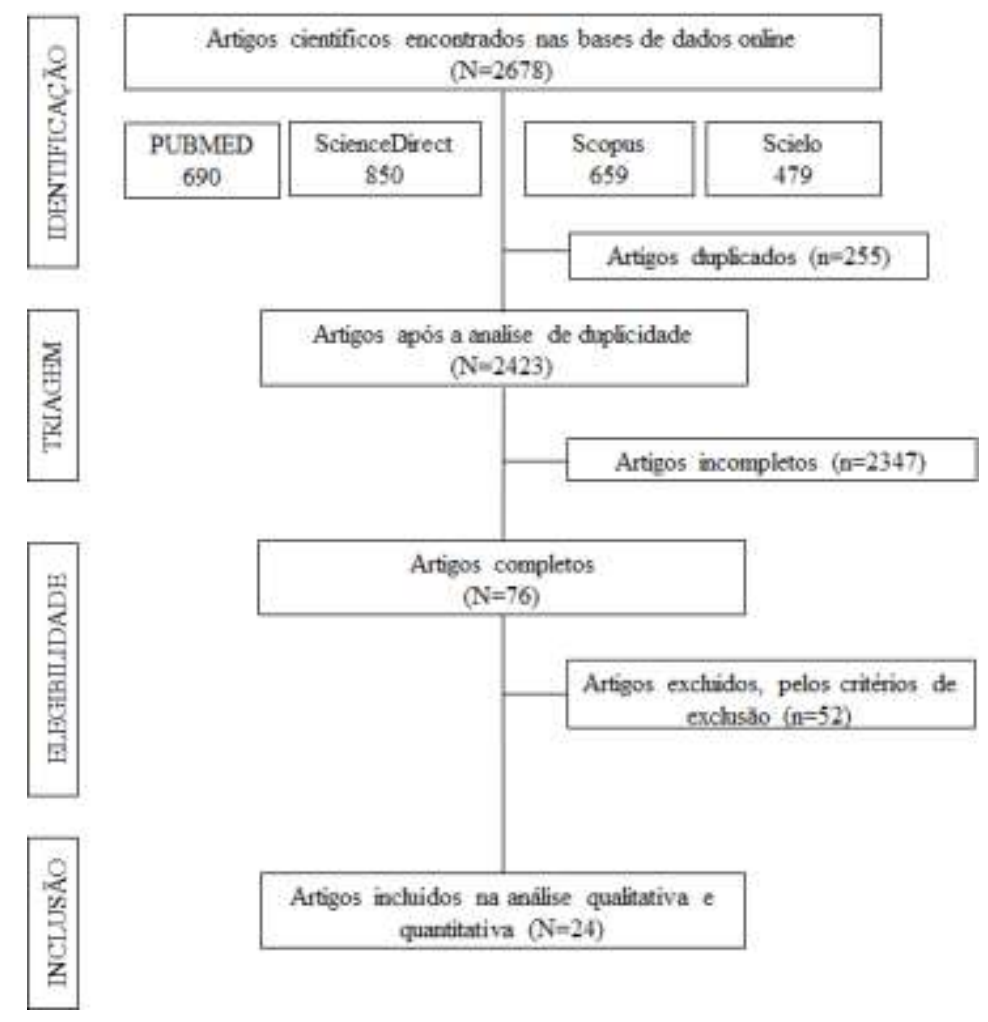

Fonte: os autores, 2021.

\subsection{Sífilis Primária}

A sífilis primária é caracterizada pelo cancro que se desenvolve nas áreas de inoculação, evidenciado, clinicamente, de 3 a 90 dias após a exposição ao Treponema Pallidum. A lesão apresenta-se como única, geralmente com presença de ulceração central, com transudado amarelado e margens altas endurecidas, sem sintomatologia dolorosa. Juntamente, edema e eritema são achados frequentemente observados (Matias et al., 2020).

O local da manifestação da sífilis primária é, normalmente, no lábio superior para homens e, em mulheres, acomete, habitualmente, o lábio inferior. Nesse estágio, quando a lesão não é tratada, o microrganismo infeccioso se dissemina, iniciando a sífilis secundária (Medoro \& Sánchez, 2021). 


\subsection{Sífilis Secundária}

Com a ocorrência do microrganismo nos vasos linfáticos, os sinais e sintomas apresentam-se de forma sistemática, como dor de garganta, mal-estar, febre e perda de peso (Farmkiss et al., 2021). O sinal mais consistente para o diagnóstico dessa fase da doença, consiste na presença de erupção cutânea indolor na região palmo-plantar. Esse estágio da doença surge a partir da quarta semana após a infecção inicial ou, em alguns casos, pode surgir antes da resolução da fase primária (Kellermann et al., 2020).

$\mathrm{Na}$ cavidade oral, a sífilis secundária apresenta-se como lesões múltiplas dolorosas, podendo ser acompanhadas de erupção cutânea. As lesões mais comuns envolvem eritema e manchas esbranquiçadas na mucosa que, ocasionalmente se unem até formar um padrão sinuoso ou semelhante a um caminho de caracol. Acometem, majoritariamente, a língua lábios, mucosa jugal e palato (Silva et al., 2020).

O diagnóstico diferencial para ulcerações encontradas no lábio deve incluir úlceras traumáticas líquen plano, eritema multiforme, quielite actínica e carcinoma de células escamosas (Lafetá et al., 2016).

Nesse estágio, a doença apresenta-se altamente contagiosa (Ikuta et al., 2021). Quando não é diagnosticada as lesões podem desaparecer dentro de 3 a 12 semanas e os pacientes podem entrar em uma fase latente da doença, com possibilidade de ocorrer recidivas no ano seguinte (Benhaddou-Mihoubi et al., 2021).

\subsection{Sífilis Terciária}

A sífilis terciária é o estágio mais grave e ocorre de um a trinta anos após a infecção inicial em pacientes que não receberam tratamento nos estágios primários e secundários (Adawaye et al., 2021). Pode haver comprometimento do sistema nervoso central, sistema cardiovascular, pele e mucosa. Os achados marcantes dessa fase são os focos dispersos de inflamação granulomatosa (chamado de goma), que se apresenta como uma lesão endurecida, nodular ou ulcerada, com risco de destruição tecidual (Seibt \& Munerato, 2016).

Habitualmente, quando a lesão se manifesta no palato, há ulceração profunda que, frequentemente, perfura em direção à cavidade nasal. Os achados clínicos são voz anasalada e comunicação bucosinusal com comprometimento na deglutição (Peng et al., 2021). Quando há acometimento da língua, ela se apresenta aumentada e com formato irregular, sendo chamada de glossite intersticial. Ainda na língua, pode haver a atrofia das papilas gustativas, reconhecida como glossite luética (Hertel et al., 2014).

\subsection{Sífilis congênita}

É causada por transmissão vertical, na qual o microrganismo atravessa a placenta da mãe, atingindo o feto. A contaminação nos dois primeiros estágios leva, na maioria dos casos, ao aborto, natimorto ou crianças com má formações congênitas (Zhang et al., 2020).

Crianças infectadas manifestam o sintoma a partir do nascimento, como prejuízo em seu desenvolvimento, febre, icterícia, anemia, rinite, entre outros (Towns et al., 2021). As crianças não tratadas que sobrevivem evoluem para a sífilis latente e posteriormente para a sífilis terciária, com danos ao ossos, dentes, olhos, orelhas e cérebro (Moccelin et al., 2020).

A infecção altera a formação dos incisivos (incisivos de hutchinson) e molares (molares em amora). A tríade de hutchinson envolve achados patognomônicos em crianças com sífilis congênita, sendo eles: dentes de hutchinson, ceratite ocular intersticial e surdez associada ao comprometimento do oitavo par de nervos cranianos. Outros sinais envolvem a presença de bossa frontal, palato arqueado, hidrocefalia, gomas e nariz em cela (Santos et al., 2019). 


\section{Considerações Finais}

A partir das trajetórias bibliográficas dos pesquisadores, esse estudo abordou as manifestações orais decorrentes da contaminação pelo Treponema Pallidum, abordando seus conceitos, características clínicas e medidas terapêuticas.

As manifestações orais para a sífilis congênita são desafiadoras para o diagnóstico. É importante que os cirurgiões dentistas saibam atuam quando estiverem presente estes tipos de lesões, reconhecendo seu estágio, direcionando ao tratamento correto, a fim de melhorar a qualidade de vida desses pacientes. Também deve ser orientado a respeito do uso de proteção nas relações sexuais, uma vez que esse é o principal meio de transmissão.

Com isso, o levantamento bibliográfico sobre as manifestações orais decorrentes sífilis se mostra importante, pois envolve estudos retrospectivos que possuem relação com o tema proposto e, ao mesmo, tempo, cria-se a possibilidade futura de novos pesquisadores abordarem o tema, criando novos trabalhos, para que haja diminuição na incidência de infectados pelo $T$. Pallidum, bem como melhoria no diagnóstico e tratamento dos pacientes infectados.

\section{Referências}

Adawaye, C., Souleymane, A. O., Fouda, A. A., Djarma, O., Cournil, A., Tuaillon, E., \& Mennechet, F. J. D. (2021). Syphilis diagnosis and serological response to Benzathine Penicillin G among patients attending HIV clinics in N'Djaména, Chad. International Journal of Infectious Diseases, 108, 461-464. https://doi.org/https://doi.org/10.1016/j.ijid.2021.05.051

Benhaddou-Mihoubi, N., Grange, P., \& Dupin, N. (2021). La syphilis : Une infection toujours d'actualité. Revue Francophone Des Laboratoires, 2021(530), 38-47. https://doi.org/https://doi.org/10.1016/S1773-035X(21)00068-X

De Andrade, R. S., De Freitas, E. M., Rocha, B. A., Da Silva Gusmão, E., Filho, M. R. M., \& Martelli, H. (2020). Oral Findings In Secondary Syphilis. Oral Surgery, Oral Medicine, Oral Pathology and Oral Radiology, 129(1), e44. https://doi.org/https://doi.org/10.1016/j.oooo.2019.06.144

Farmkiss, L., Shadrick, V., \& Bracey, T. (2021). Condylomata lata of the oral commissure: an unexpected presentation of secondary syphilis. Diagnostic Histopathology, 27(5), 226-229. https://doi.org/https://doi.org/10.1016/j.mpdhp.2021.02.007

Fregnani, E. R., Pérez-de-Oliveira, M. E., Parahyba, C. J., \& Perez, D. E. da C. (2017). Primary syphilis: An uncommon manifestation in the oral cavity. Journal of the Formosan Medical Association, 116(4), 326-327. https://doi.org/https://doi.org/10.1016/j.jfma.2016.07.004

Hertel, M., Matter, D., Schmidt-Westhausen, A. M., \& Bornstein, M. M. (2014). Oral Syphilis: A Series of 5 Cases. Journal of Oral and Maxillofacial Surgery, 72(2), 338-345. https://doi.org/https://doi.org/10.1016/j.joms.2013.07.015

Ikuta, T., Abe, S., Suga, S., Nakasone, R., Ashina, M., Tanimura, K., Nozu, K., \& Fujioka, K. (2021). Administration of intravenous benzylpenicillin in 13 infants born to mothers with syphilis infection: A case series. Journal of Infection and Chemotherapy, 27(11), 1662-1664. https://doi.org/https://doi.org/10.1016/j.jiac.2021.06.022

Kellermann, M. G., Soutto-Mayor, F., Tesseroli, M. A. S., Menegassi, J., Neto, O. V. Z., \& Guollo, A. (2020). Oral Primary Syphilis Mimicking A Gingival Squamous Cell Carcinoma: Case Report. Oral Surgery, Oral Medicine, Oral Pathology and Oral Radiology, 129(1), e121-e122. https://doi.org/https://doi.org/10.1016/j.oooo.2019.06.529

Lafetá, K. R. G., Martelli Júnior, H., Silveira, M. F., \& Paranaíba, L. M. R. (2016). Sífilis materna e congênita, subnotificação e difícil controle. Revista Brasileira de Epidemiologia, 19(1), 63-74. https://doi.org/10.1590/1980-5497201600010006

Matias, M. D. P., Jesus, A. O. de., Resende, R. G., Caldeira, P. C., \& Aguiar, M. C. F. de. (2020). Diagnosing acquired syphilis through oral lesions: the 12 year experience of an Oral Medicine Center. Brazilian Journal of Otorhinolaryngology, 86(3), 358-363. https://doi.org/https://doi.org/10.1016/j.bjorl.2018.12.010

Medoro, A. K., \& Sánchez, P. J. (2021). Syphilis in Neonates and Infants. Clinics in Perinatology, 48(2), 293-309. https://doi.org/https://doi.org/10.1016/j.clp.2021.03.005

Moccelin, M. M., Mazur, C. E., Ferreira, E. R., Veronezi, A. N. A. F. G., Torres-Pereira, C. C., Schussel, J. L., \& Amenábar, J. M. (2020). Oral Manifestations Of Secondary Syphilis. Oral Surgery, Oral Medicine, Oral Pathology and Oral Radiology, $129(1)$, e37. https://doi.org/https://doi.org/10.1016/j.oooo.2019.06.108

Peng, X., Yu, S., Wu, F., Yang, J., Wang, Y., Li, Y., Zhang, G., Lai, C., Wei, Z., \& Zhang, W. (2021). Syphilis infection and epidemiological characteristics in Haidian District, Beijing, China, 2013-2018. Public Health, 190, 62-66. https://doi.org/https://doi.org/10.1016/j.puhe.2020.11.009

Pereira, A., Shitsuka, D., Parreira, F., \& Shitsuka, R. (2018). Método Qualitativo, Quantitativo ou Quali-Quanti. In Metodologia da Pesquisa Científica. https://repositorio.ufsm.br/bitstream/handle/1/15824/Lic_Computacao_Metodologia-Pesquisa-Cientifica.pdf?sequence=1. Acesso em: 28 março 2020.

Santos, E. S., Sá, J. de O., \& Lamarck, R. (2019). Manifestações orais da sífilis: revisão sistematizada de literatura. Archives of Health Investigation, 8(8), 413-416. https://doi.org/10.21270/archi.v8i8.3330

Schuch, L. F., da Silva, K. D., de Arruda, J. A. A., Etges, A., Gomes, A. P. N., Mesquita, R. A., Vasconcelos, A. C. U., \& Tarquinio, S. B. C. (2019). Forty 
Research, Society and Development, v. 10, n. 12, e585101220943, 2021

(CC BY 4.0) | ISSN 2525-3409 | DOI: http://dx.doi.org/10.33448/rsd-v10i12.20943

cases of acquired oral syphilis and a review of the literature. International Journal of Oral and Maxillofacial Surgery, 48(5), 635-643. https://doi.org/https://doi.org/10.1016/j.ijom.2018.10.023

Seibt, C. E., \& Munerato, M. C. (2016). Secondary syphilis in the oral cavity and the role of the dental surgeon in STD prevention, diagnosis and treatment: a case series study. The Brazilian Journal of Infectious Diseases, 20(4), 393-398. https://doi.org/https://doi.org/10.1016/j.bjid.2016.03.008

Silva, E., De Andrade Munhoz, E., De Camargo, A. R., Lisboa, M. L. U. Z., I-Ching, L. E. E., Somacal, L. F., \& Grando, L. J. (2020). Oral Manifestation Of Syphilis: An Unusual Presentation Of Tertiary Syphilis. Oral Surgery, Oral Medicine, Oral Pathology and Oral Radiology, 129(1), e120. https://doi.org/https://doi.org/10.1016/j.0ooo.2019.06.519

Towns, J. M., Leslie, D. E., Denham, I., Wigan, R., Azzato, F., Williamson, D. A., Lee, D., Chow, E. P. F., Fairley, C. K., Graves, S. R., Zhang, L., \& Chen, M. Y. (2021). Treponema pallidum detection in lesion and non-lesion sites in men who have sex with men with early syphilis: a prospective, cross-sectional study. The Lancet Infectious Diseases, 21(9), 1324-1331. https://doi.org/https://doi.org/10.1016/S1473-3099(20)30838-0

Zhang, W., Mao, Q., Lyu, X., Hua, H., \& Yan, Z. (2020). Diagnosis of oral syphilis remains a challenge - A case report. International Journal of Infectious Diseases, 99, 231-232. https://doi.org/https://doi.org/10.1016/j.ijid.2020.07.049 